\title{
Relecture pragmatique de Kripke pour une approche dialogique du nom propre
}

\author{
Cormier, Agathe \\ Université Paris Ouest Nanterre La Défense \\ MoDyCo \\ thega12000@yahoo.fr
}

La théorie de Kripke est aujourd'hui largement intégrée à l'analyse linguistique, aussi bien dans les monographies concernant spécifiquement le nom propre que dans les dictionnaires de sciences du langage, les grammaires ou les manuels. Le terme de désignateur rigide, que l'on doit au philosophe, apparait en effet comme le meilleur moyen de qualifier les noms propres, qui semblent s'opposer fondamentalement aux noms communs par trois aspects : la référence unique, l'absence de sens et la stabilité de la référence. Ainsi, la Grammaire d'aujourd'hui affirme que, contrairement aux noms communs, définissables à la fois en extension et en intension, « les noms propres ont bien une extension, mais pas d'intension : ils n'ont pas, à proprement parler, de sens; seul demeure le lien qui les attache à un référent unique » (Arrivé et alii, $1986: 417)$. Et, reprenant plus loin la formule de Kripke, les linguistes précisent que « [1] es logiciens contemporains voient dans le nom propre un désignateur rigide, c'est-àdire un terme qui désigne le même individu dans tous les mondes possibles » $(1986: 417$, les auteurs soulignent). De même, la Grammaire méthodique du français remarque en premier lieu, dans son article concernant les noms propres, qu'ils «semblent [...] dépourvus de sens lexical» (Riegel et alii, 2009 : 335) ; elle ajoute qu'ils « sont cognitivement stables, puisqu'ils désignent directement leur porteur, indépendamment des variations qu'il peut subir ou des situations où il se trouve engagé ", et précise immédiatement entre parenthèses : "d'où l'appellation de "désignateur rigide" en philosophie du langage » (2009:336-337, les auteurs soulignent). Dans son manuel de pragmatique appliquée à l'analyse littéraire, Gouvard (1998) remarque aussi que la « rigidité du nom propre », par laquelle celui-ci est associé systématiquement à un individu unique, «conduit à qualifier le nom propre de désignateur rigide, c'est-à-dire comme un signe linguistique qui n'a d'autre fonction que de référer directement et toujours à telle entité singulière dont il a été décidé conventionnellement qu'elle serait désignée ainsi » (Gouvard, 1998 : 63). Mais l'exemple le plus édifiant d'une telle intégration de la théorie kripkéenne à la description linguistique du nom propre se trouve sans doute dans le Dictionnaire des sciences du langage de Neveu (2004), dont l'article suivant l'entrée nom propre contient uniquement un renvoi au terme désignateur rigide.

Le nom propre est ainsi défini comme un désignateur rigide et les autres implications de la version kripkéenne de la théorie causale sont parfois négligées. Mais cette généralité masque une certaine disparité dans l'interprétation que font les linguistes de cette théorie et dans la manière dont ils s'en servent pour expliquer la référence et la signification des noms propres. Jonasson retient par exemple que le nom propre n'a pas de sens et qu'il désigne un même référent de manière stable en vertu d'une chaîne causale qui puise son origine dans un acte de nomination et qui relie l'individu désigné au nom propre. Ainsi, pour la linguiste, c'est un «mécanisme social» (1994:115) qui fonde le lien dénominatif qui associe un nom propre à un particulier, mais ce lien dénominatif est garanti par un mécanisme cognitif dans la mesure où il "se place dans le mémoire stable et pas seulement dans la communication » (1994 : 19). Kleiber puis Gary-Prieur préfèrent de leur côté voir dans la thèse de la vacuité sémantique une mise en cause de la sémantique référentielle. Ils confèrent donc un sens au nom propre tout en appuyant également leur conception de ce sens sur la théorie causale, considérée cependant dans la perspective de la communication. Gary-Prieur soutient ainsi que la théorie causale de Kripke suggère «qu'une analyse du nom propre doit se situer dans le cadre de l'énonciation » et que «l'interprétation d'un nom propre [...] dépend très étroitement du contexte dans lequel il est employé » (Gary-Prieur, 1994 : 25). C'est sous cet angle que nous envisagerons ici le sens et la référence du nom propre.

Aussi variés soient les chemins théoriques empruntés pour expliquer les phénomènes relatifs aux noms propres, ils visent principalement à rendre compte de la stabilité de leur référence et du processus 
d'interprétation que met en œuvre leur actualisation en discours. Or la stabilité, mais aussi l'unicité de la référence du nom propre, apparaissent dans une certaine mesure comme des notions relatives. D'une part, il convient de tenir compte de l'homonymie des noms propres ${ }^{1}$, c'est-à-dire du fait qu'un même nom peut être porté par plusieurs individus. D'autre part, un individu peut changer de nom ou porter plusieurs noms différents. De plus, comme le remarque Gouvard, «nous connaissons tous des noms propres sans connaître les porteurs de ces noms » (1998: 61), ce qu'illustrent à merveille les dictionnaires des prénoms ou les atlas de noms de famille, à l'inverse des dictionnaires encyclopédiques qui représentent des nomenclatures d'individus célèbres. Par ailleurs, il existe une quantité d'emplois du nom propre, généralement qualifiés d'emplois modifiés ou non prototypiques, dans lesquels la fonction du nom n'est pas (uniquement) de faire référence au porteur de ce nom. Enfin, l'idée qu'un nom propre réfère de manière stable au même individu unique pose une question épistémologique fondamentale : celle de la définition du terme d'individu, qui apparaît souvent comme un terme primitif dans les théories de la référence des noms propres alors qu'il implique une réflexion ontologique complexe. Russell (voir 1948 : 113) propose par exemple de considérer qu'un individu n'est pas une entité simple et unique mais une série de faits liés entre eux par des relations causales. Dans cette perspective, l'affirmation selon laquelle un nom propre réfère de manière stable au même individu suppose qu'on soit en mesure de déterminer en quoi cet individu est constamment le même, et même s'il l'est.

La stabilité de la référence d'un nom propre ne semble effective que dans un cadre discursif précis et déterminé, qui implique plusieurs paramètres, notamment celui de l'identité des interlocuteurs et de leur connivence. Mais sur quoi porte cette connivence ? Sur le référent à associer au nom propre. La question est donc de savoir sur quoi repose la détermination du référent, c'est-à-dire la convention de dénomination qui associe tel nom à tel individu. L'idée de stabilité de la référence repose de plus sur la notion cognitive de mémoire à long terme et pose la question de la théorie de l'esprit mise en œuvre par les interlocuteurs. La manière dont un sujet parlant se représente les états mentaux d'un autre sujet parlant contribue certes au discours et à l'interaction en général mais, indépendamment du fait que la mémoire est faillible, autant que notre capacité à nous représenter et à exploiter avec justesse les états mentaux d'autrui, ces notions psychologiques ne rendent pas compte des phénomènes relatifs à la signification des noms propres dans une perspective linguistique. La question se posera donc également de savoir comment représenter ces paramètres de la mémoire et de la théorie de l'esprit en termes proprement linguistiques.

Il faut remarquer enfin que l'une des particularités du nom propre est d'être le fruit d'une convention explicite - même si l'acte de nomination qui fixe cette convention est «parfois perdu dans la nuit des temps» (Riegel et alii, 2009:336) - à la différence des autres signes linguistiques qui résultent de conventions tacites et implicites. L'usage d'un nom propre est en effet le produit d'un acte de nomination, ce que Kripke souligne avec force, c'est-à-dire d'un acte de parole, autrement dit d'un énoncé.

\section{Référent et porteur du nom}

Lorsqu'on s'intéresse au nom propre, on se trouve globalement confronté à deux types d'emplois : ceux où le nom réfère à l'individu auquel il est conventionnellement associé, comme dans Paul est parti à la pêche ou Frère Jacques dormez-vous?, et ceux où il renvoie à autre chose qu'à ce qu'on présume être son porteur, comme dans J'ai connu une Minville, il y a longtemps ou Quelques petits Davids vont finir par vaincre un Goliath qu'ils combattent depuis 21 ans (ces deux derniers exemples sont empruntés à Jonasson, 1994 : 11). Une distinction s'impose donc d'emblée entre le référent d'un nom propre, qui est le segment de réalité auquel renvoie l'énonciation actuelle du nom, et le porteur de ce nom, dans la mesure où référent et porteur du nom peuvent ne pas coïncider.

Cette distinction implique qu'on admette que l'usage d'un nom propre dépend essentiellement d'une convention de dénomination qui prend son origine dans un acte de nomination qui fixe un lien durable entre tel nom et tel individu. Le rôle de cet acte de nomination dans la référence et la signification des noms propres constitue précisément l'objet du présent article, mais il pourra être formulé plus clairement si la distinction entre référent et porteur du nom est déjà nettement établie. 
Le porteur d'un nom propre est l'individu auquel est attribué ce nom au cours d'un acte de nomination. C'est le référent auquel un énoncé de nomination attribue un nom, le « référent initial » du nom, comme l'appelle Gary-Prieur (1994). L'idée que nous voudrions développer, et que nous reprenons à Kripke, est que la détermination du référent actuel d'un nom propre renvoie à cet acte de nomination logiquement antérieur et fondateur. Autrement dit, l'interprétation d'un énoncé actuel contenant un nom propre renvoie à un énoncé antérieur. La détermination du référent du nom dans l'énoncé actuel implique ainsi de prendre en considération deux actes de référence distincts : celui qu'effectue l'énoncé actuel et celui qu'effectue l'acte de nomination, auquel le premier est directement relié - on verra comment plus loin. La distinction entre référent et porteur permet d'éviter de confondre ces deux aspects de la signification du nom propre, et de distinguer le rôle de l'énonciation et celui de la convention de dénomination dans la détermination de la référence du nom, c'est-à-dire de distinguer la dimension historique et la dimension subjective de l'interprétation du nom.

Mais cette distinction pose alors la question de la valeur du nom propre dans un énoncé performatif de nomination. Dans la mesure où un tel énoncé effectue l'acte de nomination qui associe initialement à un individu un nom nouveau, l'interprétation du nom propre ne peut y être renvoyée à un énoncé antérieur puisqu'il n'y a logiquement pas d'énoncé antérieur. Ce problème sera précisément développé plus loin.

Il convient par ailleurs de remarquer que si l'emploi d'un nom propre présuppose un acte de nomination logiquement antérieur, le fait que les partenaires de l'interaction connaissent l'existence de cet acte n'est pas pour autant garanti, ni même nécessaire. Autrement dit, le locuteur ni l'allocutaire ne sont obligés, avant d'employer un nom, de connaître le lien de dénomination qui le relie à son porteur, ils ne sont pas même obligés de connaître le porteur ni le nom pris séparément. Et l'interprétation du nom n'est pas pour autant mise en échec. Prenons par exemple l'énoncé :

\section{(1) J'ai vu Pierre aujourd'hui.}

D'une part, l'allocutaire peut ignorer le lien de dénomination qui relie Pierre à Pierre et même ignorer qui est Pierre ; il peut alors répondre : C'est qui, Pierre? Mais il peut aussi se contenter de sa perception du contexte pour déterminer le référent du nom Pierre. Ainsi, l'énoncé (1) dispose que Pierre est un être que le locuteur a vu le jour où il produit l'énoncé, et cette information minimale donnée par le cotexte suffit déjà à interpréter sommairement le nom propre.

D’autre part, même si cela paraît périlleux, le locuteur peut aussi de son côté employer le nom Pierre au mépris du lien dénominatif qui le relie à son porteur. Il peut ainsi, par ignorance ou par étourderie, se tromper de nom, par exemple dire Pierre en pensant à Paul, ou se tromper de personne et penser avoir vu Pierre alors que c'est Paul qu'il a vu. La communication n'est pas pour autant mise en échec, et l'allocutaire peut parfaitement comprendre de quoi veut parler le locuteur, moyennant éventuellement quelque ajustement de la référence à travers des réactions du type : Tu veux dire Paul?

Si la connaissance du lien de dénomination, c'est-à-dire de l'acte de nomination, n'est pas nécessaire à l'interprétation d'un nom propre, c'est précisément parce que l'acte de nomination est présupposé par l'emploi de ce nom; autrement dit, l'énoncé (1), en même temps qu'il désigne un référent par le nom Pierre, associe ce nom à ce référent, qui se trouve ainsi nommé Pierre. Un énoncé contenant un nom propre présuppose donc l'équivalence entre référent et porteur du nom, puisqu'il effectue implicitement un acte de nomination. Pourtant il y a de nombreuses situations où le référent n'est pas le porteur du nom et où la communication n'en est pas moins réussie. L'ambivalence entre ces deux tendances s'explique par le fait que le référent actuel d'un nom propre est déterminé par comparaison avec le porteur de ce nom, que ce référent soit ou pas le porteur du nom.

En d'autres termes, un nom propre entretient à la fois une relation actuelle avec son référent et une relation historique avec son porteur. Et en effet, la distinction entre référent et porteur du nom s'impose également parce qu'un nom propre n'est pas seulement un signe linguistique mais aussi une propriété de l'individu qu'il dénomme. Autrement dit, la convention de dénomination qui associe un nom propre à un individu est moins linguistique que culturelle et sociale. 


\section{Rôle de l'énonciation dans l'institution et l'interprétation des noms propres}

\subsection{Remarques préliminaires}

La théorie de la référence des noms propres qu'élabore Kripke dans La logique des noms propres est avant tout une théorie critique, qui se dresse contre les conceptions descriptivistes du nom propre, qu'il s'agisse de la théorie logique de Frege et Russell ou de la théorie pragmatique de Searle. Une grande partie de ses conférences se consacre donc à démontrer que la théorie descriptiviste est fausse, comme « toutes les théories philosophiques » (Kripke, $1972: 51)$.

Kripke espère d'ailleurs qu'on ne le soupçonne pas de proposer une «théorie de rechange » car il considère que « les théories philosophiques ont toutes les chances d'être fausses » (voir 1972:81) et que celle qu'il proposerait « serait fausse aussi, en tant que théorie » (1972: 51). Il se risque néanmoins à « un début de théorie », qu'il présente cependant comme une « esquisse » (1972:84), dont il répète ensuite que ce «n'est pas une théorie, mais une représentation plus conforme à ce qui se passe que celle que mettent en avant les théoriciens descriptivistes » $(1972: 86)$.

Cette approche essentiellement critique, voire sceptique, lui a d'ailleurs été reprochée par Searle, qui accuse la théorie de Kripke de ne pas se présenter «comme le résultat d'une analyse de l'emploi des noms entreprise pour des raisons indépendantes », et de n'avoir d'autre objectif que de se dresser en « concurrente de la théorie descriptiviste » (Searle, 1983 : 287). Dans la mesure où ce que propose Kripke $n$ 'est pas une théorie, l'objection reste impuissante.

L'approche kripkéenne est en effet radicalement différente en ce qu'elle envisage moins, malgré le terme de désignateur rigide, le nom propre comme une constante logique que comme un moyen de la communication, et qu'elle redéfinit la référence du nom selon son rôle dans l'échange verbal. Dès l'ouverture de ses conférences, Kripke fait ainsi une mise au point préalable sur le terme de nom: «Par un nom, j'entends ici un nom propre, c'est-à-dire le nom d'une personne, d'une ville, d'un pays, etc. » (1972 : 13). Il emploie donc le terme de nom pour désigner la catégorie lexicale des noms propres, qu'il définit d'abord en extension, en ébauchant une typologie de cette classe de mots. On peut remarquer cependant qu'il s'intéresse surtout à l'anthroponyme, érigé en quelque sorte en modèle du nom propre. Sa définition des noms propres reste relativement restrictive mais il s'intéresse résolument aux noms tels qu'on les emploie dans le langage ordinaire plutôt qu'à des termes logiques et sa définition recouvre assez bien la classe traditionnellement définie par les grammairiens.

Contrairement à Frege qui appelle «nom propre » toute "désignation d'un objet singulier », qu'elle consiste en un ou plusieurs mots, c'est-à-dire aussi bien point d'intersection de a et b, étoile du soir qu'un « nom propre véritable» comme Aristote (Frege, 1892:103-104), Kripke commence en particulier par distinguer les noms propres des descriptions définies (voir Kripke, 1972:13). Sa critique des théories descriptivistes repose d'ailleurs sur les deux distinctions parallèles suivantes : la distinction entre noms (propres) et descriptions (définies) d'une part, et l'opposition entre désignateur rigide et désignateur accidentel d'autre part.

Remarquons enfin, avant d'entrer dans ses détails, que la «théorie » de Kripke ne pose pas la notion de désignateur rigide comme le terme de son argumentation mais plutôt comme une de ses prémisses; autrement dit, l'idée que le nom propre désigne le même individu dans tous les mondes possibles, c'est-àdire qu'il est capable de désigner un même individu de manière plus ou moins univoque et stable, quelle que soit la situation réelle ou fictive considérée, n'est pas une thèse qu'il s'agirait de démontrer mais précisément ce dont il convient de rendre compte. Kripke aussi bien que les tenants des théories descriptivistes cherchent à expliquer la désignation unique et stable, mais ils empruntent des directions inverses : ceux-ci ont une approche onomasiologique, qui considère le concept de référence unique pour établir les conditions de son expression linguistique, tandis que celui-là adopte une démarche plutôt sémasiologique, qui vise à expliquer l'usage des formes linguistiques. Kripke élabore d'ailleurs davantage 
une théorie de la nomination, comme le précise le titre original de ses conférences ${ }^{2}$, qu'une théorie des noms propres.

\subsection{Relation entre noms et descriptions}

Kripke émet à l'encontre de la thèse d'un sens descriptif du nom propre deux objections liminaires :

- d'une part, dans la mesure où la signification d'un nom propre correspond à une ou plusieurs descriptions, et dans la mesure où cette ou ces descriptions sont synonymes du nom propre, un même nom peut avoir différents sens selon les locuteurs et les situations, comme le montre merveilleusement le $\S 79$ des Recherches philosophiques de Wittgenstein (1953 : 70-72).

- d'autre part, si le sens du nom consiste en une description, alors les énoncés d'identité du type Aristote fut le précepteur d'Alexandre le Grand sont tautologiques, puisque l'information donnée par le groupe verbal est censée être déjà contenue dans le sens du nom sujet Aristote.

Mais, selon Kripke, il y a deux manières d'envisager la théorie descriptiviste : premièrement, comme théorie du sens du nom propre et, dans ce cas, le sens du nom propre est constitué par un faisceau de descriptions substituable au nom propre dans une proposition sans en changer la valeur de vérité; deuxièmement, comme théorie de la référence des noms propres, selon laquelle une description, même si elle ne constitue pas le sens d'un nom propre, peut servir à en fixer la référence. Cette deuxième façon d'envisager la théorie descriptiviste reste acceptable pour Kripke.

\subsubsection{Identifier et référer}

Les tenants du sens descriptif cherchent à établir une définition analytique du nom propre en termes de propriétés nécessaires et connaissables a priori du référent, qui expliquerait, selon eux, la référence définie unique et constante des noms propres en établissant les conditions que tel individu doit remplir pour être identifié par tel nom. Le problème selon Kripke est qu'ils considèrent une définition analytique comme nécessaire et fondée a priori. Ils utiliseraient ainsi la notion de nécessité dans un sens épistémique, comme équivalent d'a priori. Les théoriciens descriptivistes confondraient de la sorte la question de savoir ce qu'on peut connaître indépendamment de toute expérience, qui relève de la théorie de la connaissance, avec la question de savoir si ce qui est vrai aurait pu être autrement, qui relève de la métaphysique et «n'a en soi rien à voir avec la connaissance qu'a quelqu'un de quelque chose » (Kripke, $1972: 24)$.

Pour Kripke, le problème des théoriciens descriptivistes réside donc, d'une part, dans la volonté d'établir une définition analytique des noms propres, c'est-à-dire une définition à la fois nécessaire et fondée $a$ priori et, d'autre part, dans la confusion entre ces deux termes.

Il remarque de plus que les propriétés singularisantes du référent d'un nom propre ne sont pas connaissables a priori et que, de toute façon, la référence peut être réussie sans qu'il soit possible pour le locuteur ou ses interlocuteurs de donner aucune propriété singularisante du référent. Il remarque, en outre, que les propriétés associables à un nom propre ne sont pas non plus en général des propriétés nécessaires de son référent.

Autrement dit, les propriétés associées à un nom ne sont, pour Kripke, ni nécessaires ni susceptibles d'être connues a priori. Mais surtout, le nom propre n'est pas pour lui un moyen d'identifier un particulier mais un moyen de référer à un particulier.

En effet, si le nom propre est un moyen d'identifier un particulier, il doit alors être défini en termes de propriétés nécessaires du référent, c'est-à-dire en termes de propriétés vraies dans tous les mondes possibles. Or, pour Kripke, « un monde possible n'est pas un pays lointain qu'on rencontre sur son chemin ou qu'on regarde au télescope », il est «donné par les conditions descriptives que nous lui associons » (1972:32, l'auteur souligne). En d'autres termes, un monde possible n'est pas un observable mais une situation contrefactuelle stipulée, dont le point de repère est le monde réel. Ou, dans les termes 
de Gary-Prieur: les mondes possibles «n'ont pas d'existence extérieure au discours qui les met en place » (1994: 21). Concrètement, lorsqu'on emploie un nom propre, on ne détermine pas son référent en cherchant quel individu répond aux propriétés associées à ce nom dans l'univers considéré mais le nom propre lui-même actualise un individu dont les propriétés dépendent de l'univers considéré. Cela permet notamment de rendre compte d'énoncés conditionnels du type Aristote aurait pu ne pas être le précepteur d'Alexandre: si dans le monde «réel» du discours Aristote est reconnu comme le précepteur d'Alexandre, dans le monde hypothétique évoqué par cet énoncé, il ne possède plus cette propriété ; le nom Aristote désigne néanmoins le même individu dans le monde «réel » et dans le monde possible stipulé, indépendamment donc des propriétés qui lui sont attribuées. C'est précisément que le rôle du nom propre n'est pas d'identifier un individu selon certaines propriétés singulières, mais de référer à un particulier.

Il n'est donc pas besoin d'identifier un objet selon certaines propriétés essentielles pour poser son existence dans tel monde possible, il suffit de nommer un objet pour en poser l'existence. En effet, les noms propres n'ont pas pour fonction d'identifier un particulier mais de référer à un particulier; ils ne décrivent pas les propriétés identifiantes du référent mais constituent l'instance d'un particulier dans le discours. Ainsi, en vertu d'une convention linguistique, tel nom réfère à tel individu quelles que soient les situations contrefactuelles dans lesquelles il est pris, c'est-à-dire quelles que soient ses propriétés. C'est en cela que le nom propre est un désignateur rigide et pas un désignateur accidentel et c'est justement là que réside l'erreur des descriptivistes, selon Kripke : en soutenant que le sens d'un nom propre consiste dans une description définie, qui ne peut jamais saisir que des propriétés contingentes du référent visé, ils font des noms propres des désignateurs accidentels, incapables de référer à un individu donné indépendamment des propriétés qui lui sont attribuées. En définitive, Kripke reproche aux théories descriptivistes d'éliminer la notion de référence, qui est pourtant une «notion primitive » à ses yeux (voir Kripke, 1972 : 85), au profit de celle d'identification. C'est pour cela qu'il considère que la conception descriptiviste comme théorie du sens des noms propres est tout simplement fausse.

\subsubsection{Théorie de la nomination}

Une description ne peut constituer le sens d'un nom propre ni être synonyme d'un nom propre, parce que les descriptions sont des désignateurs accidentels et représentent des propriétés contingentes du référent qu'elles désignent. En d'autres termes, la référence d'une description dépend de la configuration du monde, « réel » ou possible, considéré.

Au contraire, les noms propres sont des désignateurs rigides, ce qui implique, d'une part, qu'ils «n'ont pas de sens », c'est-à-dire qu'ils désignent directement un particulier, sans l'intermédiaire d'une description singularisante et, d'autre part, qu'ils désignent rigidement un particulier, c'est-à-dire nécessairement le même particulier dans tous les mondes possibles. Dans la mesure où l'acte de nomination « fixe la référence par description ou par ostension » (Kripke, 1972:85), une description peut par contre servir à fixer la référence d'un nom propre et, à ce titre, Kripke considère que la théorie descriptiviste comme théorie de la référence constitue une bonne représentation de la nomination, c'est-àdire de la manière dont un nom est conventionnellement attribué à un individu. Comme le remarque Gary-Prieur, l'acte de nomination «joue un rôle fondamental dans la conception de Kripke : un nom propre est attribué à un individu, et toute utilisation ultérieure du nom renvoie à cet acte premier » (GaryPrieur, 1994 : 19). La théorie de la référence des noms propres de Kripke est en effet avant tout une théorie de la nomination, c'est-à-dire en quelque sorte une théorie de la relation discursive et sociale entre un nom propre et son porteur. Voici l'une des formulations qu'il en donne :

Un bébé naît ; ses parents lui donnent un nom. Ils parlent de lui à leurs amis. D’autres personnes font sa connaissance. À travers des conversations de toutes sortes, le nom est transmis comme par une chaîne, de maillon en maillon. [...] [Un locuteur] est relié à une chaîne de communication à une extrémité de laquelle se trouve l'homme auquel il fait référence. (Kripke, $1972: 79$ ) 
L'emploi d'un nom propre implique donc, d'une part, un acte initial public de nomination, où la référence est fixée par description ou par ostension et, d'autre part, une communauté de locuteurs au sein de laquelle se transmet le nom à travers diverses conversations. L'acte de nomination donne ainsi naissance à une chaîne de communication qui relie les membres de la communauté de locuteurs en question et le long de laquelle se diffuse l'usage du nom. Dans cette perspective, pour faire référence à l'aide d'un nom propre, un locuteur n'a besoin de connaître aucun fait concernant le référent; participer à une situation d'énonciation où le nom est mentionné est suffisant pour en connaître l'usage, au moins rudimentaire. La référence d'un nom propre dépend donc principalement de l'interaction au sein d'un réseau de locuteurs dont les membres sont déterminés par leur relation directe ou indirecte à l'acte de nomination qui initie l'usage de ce nom.

\title{
2.3 Nomination et chaînes causales
}

Pour Kripke, donc, les noms propres ne réfèrent pas par l'intermédiaire d'un sens mais en vertu d'une convention entérinée par l'usage. Une conséquence moins acceptable de la thèse de la référence directe telle que l'envisage Kripke est que le nom propre n'a pas de sens.

Si l'on considère, à l'exemple de Saussure, qu' " [i]l n'y a aucune différence sérieuse entre les termes de valeur, sens, signification, fonction ou emploi d'une forme, ni même avec l'idée comme contenu d'une forme » et que « ces termes sont synonymes » (Saussure, $2002: 28$ ), outre l'aberration que représenterait un signe sans signifié, on ne peut raisonnablement pas admettre qu'une forme soit dépourvue de sens. De plus, même si l'on admet l'idée d'une référence directe, par exemple pour qualifier le mode de signification des embrayeurs et autres déictiques, il n'en reste pas moins que la référence de telles formes linguistiques est relative à leur emploi, leur fonction ou leur valeur, autrement dit à leur sens, quel qu'il soit. Kleiber considère ainsi qu'à l'interprétation d'un nom propre s'applique également la règle de subordination référentielle selon laquelle « la référence actuelle d'une expression dépend de sa référence virtuelle ou sens » (Kleiber, 1981:324), mais il constate cependant que le caractère non descriptif du nom propre implique une caractérisation spécifique de ce sens. Ou plus prosaïquement, la thèse de la vacuité sémantique du nom propre

\begin{abstract}
est difficilement soutenable, tout simplement parce que le porteur du nom propre n'étant jamais lui-même présent dans la phrase, il faut bien que le nom propre, s'il entend renvoyer à quelque chose d'autre que lui-même et pas à n'importe quoi, contienne des indications de quelque ordre que ce soit, qui dirigent notre interprétation vers ce pour quoi il est là. [...] Dit autrement, il faut bien qu'il y ait des conditions d'emploi, c'est-à-dire du sens. (Kleiber, 2004 : 117)
\end{abstract}

Comme le remarque Vaxelaire (voir 2005 : 719-720), il s'agit davantage pour Kripke de récuser les principes de la logique véri-conditionnelle, qui décrit le sens du nom propre en termes de conditions nécessaires et suffisantes d'identification du porteur du nom. Une description définie ne décrivant que des propriétés accidentelles de l'individu qu'elle désigne, elle ne désigne cet individu qu'accidentellement, par le biais de telles de ses propriétés potentiellement variables. Le nom propre, au contraire, désigne nécessairement le même individu quelles qu'en soient les propriétés, justement parce qu'il désigne directement l'individu en tant que tel et n'en décrit aucune propriété. Le refus d'attribuer un sens aux noms, y compris d'ailleurs aux «noms communs » ou «noms d'espèces naturelles » (voir Kripke, $1972: 115)^{3}$, consiste donc surtout dans une mise en cause de la sémantique référentielle.

À la page 65 de sa Logique des noms propres, Kripke s'explique de la manière suivante sur le terme de désignateur rigide :

Quand je qualifie un désignateur comme rigide, comme désignant la même chose dans tous les mondes possibles, je veux dire qu'en tant qu'employé dans notre langage il désigne cette chose, quand nous parlons d'une situation contrefactuelle. (1972:65)

Il nous semble qu'en soulignant la référence à la première personne (notre, nous) Kripke insiste sur les deux notions essentielles à retenir: celle de convention et celle d'usage, qui reposent sur les deux 
fondements de sa théorie de la nomination que sont l'acte de nomination et les chaînes de communication, aussi appelées chaînes causales.

La convention par laquelle un nom propre réfère rigidement à un particulier implique, on l'a vu, un acte de nomination et une communauté de locuteurs au sein de laquelle se transmet le nom. L'acte de nomination implique quant à lui les quatre paramètres suivants : un énoncé performatif qui accomplit l'acte de nomination, un locuteur habilité à le prononcer, la présence ou la présentification de la chose nommée (par ostension ou description), et des interlocuteurs témoins et garants de l'acte. On peut considérer que le locuteur de l'énoncé performatif et les interlocuteurs présents lors de l'acte de nomination constituent le noyau dur du réseau de locuteurs au sein duquel circule le nom. Ainsi l'utilisation référentielle du nom propre repose sur un acte de langage, c'est-à-dire sur un énoncé performatif qui constitue le premier emploi du nom propre et fixe le lien dénominatif entre ce nom et son porteur. À partir de cet acte fondateur de nomination se développent des chaînes de communication à travers lesquelles se déploie l'usage du nom et qui relient chaque emploi du nom propre à un de ses emplois antérieurs. Et c'est précisément ce que signifie l'idée de chaînes «causales»: chaque énonciation du nom est en quelque sorte causée, c'est-à-dire rendue possible, par une énonciation antérieure (dont la cause originelle, si l'on remonte tous les maillons de la chaîne, serait l'acte initial de nomination).

Kleiber a bien relevé cette importance de l'interaction dans la théorie kripkéenne puisqu'il souligne que « [1]a compétence référentielle est le résultat de l'interaction entre celui qui dénomme, le nom qu'il emploie, l'objet dénommé et ceux qui assistent à cette dénomination. » Mais il ajoute : "On peut donc dire que la chaîne causale prend son départ dans l'objet lui-même » (Kleiber, 1981 : 379). Alors qu'il explique que la théorie de Kripke peut être qualifiée de « causale » parce que les participants «perçoivent directement les éléments constitutifs de l'acte de dénomination » (Kleiber, 1981:379), il réduit ensuite subrepticement la perception directe des éléments de l'acte de nomination à l'identification de l'objet nommé. Or l'objet nommé en lui-même ne cause rien de spécial, c'est l'acte de nomination qui cause l'usage du nom propre. Searle avait d'ailleurs perçu cette particularité du lien causal chez Kripke :

Aussi est-ce un trait bien singulier de la version kripkéenne de la théorie causale que la chaîne causale externe ne remonte pas en fait jusqu'à l'objet, mais qu'elle remonte jusqu'au baptême de l'objet, c'est-à-dire jusqu'à la cérémonie où le nom a été mis en circulation. (Searle, $1983: 280$ )

On peut ajouter que cette notion de relation causale renvoie dans les faits à l'antériorité logique ; en effet, tout emploi d'un nom propre ne dépend pas nécessairement d'un énoncé de nomination préalable, performatif ou didactique ${ }^{4}$, mais plutôt de la possibilité d'un tel énoncé pour expliquer l'emploi du nom propre.

\subsection{Problème de l'intention}

La particularité de l'approche philosophique de Kripke, que nous voudrions ici souligner et développer en termes proprement linguistiques, est qu'en fondant sa théorie sur les notions d'acte de nomination et de chaînes de communication, il place plus ou moins implicitement l'énonciation au cœur de la description du nom propre. L'acte de nomination constitue en effet un énoncé et les maillons de la chaîne causale de communication constituent également des énoncés. La référence du nom propre est ainsi expliquée par son actualisation dans des énoncés déterminés. Il nous semble que c'est précisément la nature de cette détermination que l'analyse linguistique éprouve des difficultés à appréhender.

D'un point de vue linguistique, un énoncé est principalement déterminé par les paramètres de l'énonciation que sont le locuteur, l'allocutaire, et la situation spatio-temporelle dans laquelle a lieu l'acte d'énonciation. En d'autres termes ce sont ces paramètres - adossés à d'autres facteurs comme la (ou les) langue(s) utilisée(s) pour l'échange (à travers l'expression de ses règles), l'identité, l'intention et les connaissances des interlocuteurs ainsi que leurs relations mutuelles, etc. - qui expliquent qu'un acte d'énonciation puisse avoir lieu, qui le causent en quelque sorte, mais aussi qui expliquent l'interprétation que l'on peut en faire. Autrement dit, interviennent là des paramètres linguistiques aussi bien que 
culturels, sociaux et psychologiques. Ce qui pose deux problèmes : l'impossibilité certaine de saisir la totalité des paramètres qui déterminent un énoncé, et la difficulté à démêler et répartir nettement les facteurs observables entre les différents aspects - linguistiques, culturels, etc. - du phénomène de l'énonciation.

L'intention du sujet parlant, lorsqu'il produit (ou reçoit) un énoncé, fait partie des paramètres difficilement appréhendables d'un point de vue strictement linguistique, et néanmoins indispensables dans la compréhension des énoncés, en particulier lorsque ceux-ci usent d'ironie, de mensonge, de feinte, d'erreur, etc. On peut dire que l'intention est d'une certaine manière pour le linguiste une notion primitive, dont la définition excède les limites de la linguistique : si l'on ne postulait pas que le locuteur a l'intention de communiquer quelque chose - quoi que ce soit - il n'y aurait aucun sens à chercher des principes d'explication de la signification, quoi que l'on entende par ce dernier mot.

De même la mémoire, qui garantit des choses aussi élémentaires que la connaissance d'une langue, la reconnaissance des individus et toute une série de comportements qui participent de la communication, fait partie de ces paramètres avec lesquels le linguiste compose sans se sentir obligé de se prononcer sur leur définition. Ainsi « celui à qui le nom est transmis doit, au moment où il en prend connaissance, avoir l'intention de l'utiliser avec la même référence que l'homme dont il l'a appris » écrit Kripke (1972 : 85). Autrement dit la référence d'un nom propre implique une certaine intention - celle d'utiliser un nom avec la même référence que quelqu'un d'autre - et de posséder certaines connaissances en mémoire - celles qu'on apprend au cours d'un acte de nomination, qu'elle soit didactique ou performative. Ce sont deux aspects de la théorie de Kripke que nous avions provisoirement laissés de côtés et qu'il nous faut maintenant transposer en termes linguistiques.

On mettra ici de côté les cas d'erreur de transmission ou de référence erronée - surtout parce qu'on serait alors redevable d'une définition précise de ce qu'on appelle erreurs ou fautes de référence - pour s'intéresser au cas, pour ainsi dire, normal. Il nous semble en effet que l'idée d'avoir l'intention d'utiliser un nom avec la même référence répond justement à une norme culturelle et sociale qui contribue à régler l'usage linguistique du nom, à une norme à la fois comportementale et morale qui exige que lorsqu'on apprend le nom de quelque individu, on s'efforce de l'utiliser correctement, c'est-à-dire conformément à l'acte de nomination qui a fixé le lien de dénomination entre ce nom et cet individu. C'est seulement à condition que cette norme soit respectée que l'on pourra considérer que l'acte de nomination, en tant qu'acte de langage, est réussi. Concrètement, les sujets parlants présents lors de l'acte de nomination initial s'engagent à apprendre, appliquer, reproduire et enseigner l'usage qui consiste à référer à tel individu à l'aide de tel nom, précisément parce que l'usage d'un nom propre est le fruit d'une convention explicite, même si son origine se perd dans la nuit des temps.

Notre tâche est donc ici de transposer en termes linguistiques les notions d'intention et de mémoire qui, corrélées aux paramètres de l'acte de nomination et aux chaînes causales de communication, constituent les fondements de la référence des noms propres. Il nous semble que ces deux notions peuvent s'exprimer en termes de théorie des actes de langage ${ }^{5}$. Utiliser un nom propre consiste en effet à suivre une convention de dénomination - ou à l'enfreindre, que ce soit délibérément ou involontairement - ; il convient donc de rendre compte des conditions dans lesquelles une telle convention peut être établie.

Lorsqu'un sujet parlant assiste ou participe à un acte de nomination didactique ou performative, s'il joue le jeu, il a en quelque sorte le devoir de se souvenir du nom impliqué, de l'individu qui le porte et du lien dénominatif qui les relie. Le lien dénominatif entre un nom propre et un individu apparaît ainsi comme un effet pragmatique de l'acte de nomination : l'énoncé performatif qui le réalise établit d'une part le lien dénominatif entre ce nom et cet individu (c'est là sa force illocutoire), et oblige d'autre part les interlocuteurs à utiliser la règle ainsi établie dans leurs échanges ultérieurs (c'est là sa force perlocutoire). L'intention d'utiliser la même référence et la mémoire du lien dénominatif sont ainsi conçues comme des effets pragmatiques de l'acte de nomination. Et c'est à condition que l'acte de nomination remplisse pleinement sa fonction performative que le nom propre devient un désignateur rigide. La désignation rigide est donc l'effet pragmatique attendu d'un acte de nomination, une sorte d'obligation morale qui n'a d'autre but métaphysique que d'assurer l'intercompréhension. 
Mais l'intention qui détermine l'usage d'un nom propre et la mémoire qui le garantit ne se laissent pas entièrement réduire aux effets pragmatiques de l'acte de nomination qui initie cet usage; encore faut-il rendre compte d'un point de vue linguistique de la transmission de l'usage du nom propre et du déploiement du réseau de communication qui le véhicule. Ou plutôt, l'invocation de la théorie des actes de langage explique la production d'un énoncé contenant un nom propre mais reste évasive sur son interprétation. Autrement dit, maintenant qu'on a expliqué quel parcours conduit de l'acte de nomination à l'énonciation actuelle du nom propre, il convient de parcourir le chemin inverse, pour expliquer quel phénomène permet de remonter jusqu'à l'acte de nomination initial.

\section{Le nom propre comme unité dialogique}

Dans la mesure où la référence actuelle d'un nom propre est à la fois déterminée par un acte de nomination qui fixe un lien de dénomination reliant ce nom propre à un individu particulier, et par une chaîne de communication reliant l'acte de nomination à l'énoncé actuel, l'interprétation d'un nom propre renvoie à un emploi logiquement antérieur de ce nom; autrement dit, l'interprétation d'un énoncé contenant un nom propre renvoie à un voire plusieurs énoncés autres : à des énoncés comme T'as vu Paul hier? ou Tiens, Paul, t'es là?, mais aussi des énoncés comme C'est Paul, Il s'appelle Paul ou Il s'appellera Paul, et toutes sortes d'autres énoncés innombrables qu'il serait vain de vouloir répertorier. Ainsi la référence actuelle du nom propre dépend de la transmission du nom le long de ces chaînes de communication qui tissent le réseau de locuteurs indéfini au sein duquel circule le nom. Le nom se transmet d'énoncé en énoncé, chaque énoncé prolongeant la chaîne d'un maillon. L'énoncé actuel renvoie à un énoncé antérieur, qui renvoie lui-même à un énoncé antérieur, qui lui-même renvoie à un autre énoncé, et ainsi de suite jusqu'à l'acte de nomination.

L'expression d'un énoncé est toujours, à un degré plus ou moins grand, une réponse, autrement dit : elle manifeste non seulement son propre rapport à l'objet de l'énoncé, mais aussi le rapport du locuteur aux énoncés d'autrui. (Bakhtine, 1979 : 299)

C'est ainsi que Bakhtine introduit la notion de dialogisme dans l'analyse linguistique. On ne peut alors s'empêcher de concevoir le nom propre comme l'une des « harmoniques dialogiques [qui] remplissent un énoncé » (Bakhtine, 1979 : 300). Selon l'analyse que l'on vient d'en faire, l'énonciation d'un nom propre est une sorte de réponse à une énonciation antérieure de ce nom. Autrement dit, il semble que le nom propre soit, dans le fonctionnement même de sa référence, une unité dialogique ; ou, comme le dit Siblot, le dialogisme se trouve « au cœur du nom » $(1997: 55)$.

Par ailleurs, nous avons vu plus haut qu'il est inconcevable de refuser un sens au nom propre. La question qui se pose alors est de savoir quelle expression du sens permet de rendre compte de ce fonctionnement dialogique du nom propre.

\subsection{Formalisation linguistique de la théorie de Kripke}

\subsubsection{Un sens dénominatif}

La thèse du sens dénominatif de Kleiber nous semble constituer une bonne réponse à cette question, moyennant quelques précisions et adaptations à la théorie kripkéenne. Kleiber adopte d'ailleurs les mêmes prémisses dans son analyse de la référence des noms propres. La théorie du sens dénominatif qu'il développe répond ainsi à deux impératifs : formuler le sens du nom propre en tenant compte de son caractère non descriptif, et lui intégrer la présupposition de nomination préalable qu'implique l'emploi d'un nom propre.

Alors que Kleiber avait défini en 1981 le nom propre comme un prédicat de dénomination, la formulation la plus aboutie de sa théorie s'affirme dans des articles plus récents. Renonçant à l'option prédicative pour diverses raisons qu'il n'est pas le lieu ici de développer (voir Kleiber, 1995 et 2004), Kleiber propose de concevoir le sens dénominatif comme correspondant à «l'instruction de chercher ou de trouver dans la 
mémoire stable le référent qui porte le nom en question » (2004:131), autrement dit, de doter les noms propres d'un sens instructionnel ou procédural (voir 2004 : 131), à l'image des embrayeurs, déictiques ou autres symboles indexicaux.

\begin{abstract}
En prenant modèle sur l'appellation symbole indexical, où le premier terme indique qu'il s'agit d'expressions ayant un sens conventionnel, stable, et la deuxième quelle est la procédure indiquée par ce sens, on peut qualifier les noms propres de symboles dénominatifs : ils sont à la fois des symboles, parce qu'ils ont un sens conventionnel et ce sont des marqueurs dénominatifs, parce que ce sens invite à retrouver en mémoire stable le référent porteur de ce nom. (Kleiber, 2004 : 132)
\end{abstract}

La procédure indiquée par le nom propre pour en déterminer le référent correspond donc à l'instruction de “chercher le référent $x$ qui porte le nom $N$ ". Chercher en mémoire stable, c'est-à-dire aussi dans le contexte situationnel. Autrement dit, l'application de la procédure de détermination du référent implique aussi bien de prendre en compte l'identité des interlocuteurs que la configuration pragmatique de la situation de l'énonciation et ses différents paramètres.

Les éléments du contexte pertinents pour l'interprétation sont plus ou moins variables selon les situations, mais dans la mesure où la notion de sujet implique celle d'intersubjectivité, et dans la mesure où les données contextuelles s'organisent autour du sujet, on peut, afin de marquer le rôle des interlocuteurs c'est-à-dire leur intention - et de la situation dans le sens même du nom propre, rassembler les différents paramètres du contexte sous le terme de sujet. Ce terme peut être représenté par $S$ et ajouté à la formulation du sens instructionnel dénominatif, qui peut ainsi devenir "chercher le référent $x$ dénommé $N$ par $S$ ", où $x$ représente le référent, $N$ le nom propre et $S$ le sujet de l'énonciation.

\title{
3.1.2 Objections et concessions de Kripke
}

Il se trouve que Kripke a examiné - rapidement, au détour d'une réflexion sur la condition de noncircularité que toute théorie est censée remplir pour éviter l'absurdité et la régression à l'infini des explications issues de ses thèses - la théorie de Kneale selon laquelle « le mot "Socrate" [...] signifie "l’individu appelé Socrate” » (Kneale, 1962: 630). Le logicien infère cette affirmation de l'idée qu'il serait trivial de dire que Socrate s'appelait Socrate : cette information n'a pour lui aucune pertinence dans la mesure où on ne peut employer le nom Socrate sans présupposer qu'il réfère à un individu appelé Socrate. L'énoncé :

\section{(2) Socrate s'appelle Socrate}

serait donc trivial, c'est-à-dire que son explication serait circulaire, parce que le nom Socrate signifie déjà "l'individu appelé Socrate" : l'énoncé (2) n'apporte en effet dans cette perspective aucune information nouvelle puisque la référence du nom Socrate est déterminée par renvoi à l'individu appelé Socrate, ce qui signifie qu'en expliquant la première mention du nom Socrate dans cet énoncé on fait appel au reste de l'énoncé. En d'autres termes, le prédicat de cette phrase est déjà contenu dans le sujet.

Pour Kripke cependant, un énoncé comme (2) n’est pas nécessairement trivial. Ce qui est pour lui en revanche « ouvertement circulaire» (Kripke, 1972:59), c'est la théorie selon laquelle le nom Socrate signifie "l'individu appelé Socrate".

Si quelqu'un cherchait à déterminer pour lui-même le référent d'un nom comme «Glunk » et prenait la décision suivante : «J'emploierai le terme "Glunk" pour faire référence à l'homme que j'appelle "Glunk" », cela ne le mènerait nulle part. Il ferait mieux de disposer d'un moyen indépendant de déterminer le référent de « Glunk ». C'est là un bon exemple de détermination manifestement circulaire. (Kripke, $1972: 60-61$ )

L'exemple de Kripke est en effet aussi comique qu'absurde, et ne donne aucune indication sur la manière de déterminer la référence du nom Glunk. Mais il y a un moyen de déterminer le référent qui s'en approche beaucoup et qui ne nécessite qu'une légère modification de cet exemple. Le problème que soulève Kripke est à notre avis résolu si l'on remplace l'individu que j'appelle "Glunk » par l'individu 
qu'on appelle "Glunk», c'est-à-dire si on introduit une différence de portée dans la convention de dénomination énoncée par ce segment. Autrement dit, il existe un bon moyen de déterminer quel individu s'appelle Glunk, c'est d'observer quel est l'individu, dans mon entourage direct ou indirect, qu'on appelle Glunk. Si des doutes persistent, notamment quant à savoir quels locuteurs je juge les plus dignes de ma confiance, je peux évaluer de diverses manières ceux qu'il me paraît bon de suivre comme modèles. C'est que l'usage des noms propres est principalement déterminé par une convention sociale - fixée par un acte de nomination - qui n'établit une ligne de conduite que pour autant qu'elle est effectivement appliquée.

Autrement dit, convention et usage sont deux faces d'une même pièce, et l'emploi de tel nom pour référer à tel individu est le produit de pressions morales, sociales, culturelles... ou, pour reprendre les termes de Bakhtine, une conséquence du fait d'envisager le langage non «comme un système de catégories grammaticales abstraites, mais comme un langage idéologiquement saturé, comme une conception du monde, voire comme une opinion concrète, comme ce qui garantit un maximum de compréhension mutuelle dans toutes les sphères de la vie idéologique » (Bakhtine, 1975 : 95). Nous convenons certes que l'emploi des noms propres ne représente pas à première vue un enjeu idéologique majeur, mais peut-être est-ce aussi parce que nous restons au niveau de la théorie sans entrer dans le détail d'analyses d'emplois spécifiques.

Toujours est-il que la question que se pose Kripke est de savoir comment déterminer la référence d'un nom propre, c'est-à-dire à la fois comment employer et interpréter un nom propre. Or, il nous semble que l'option dénominative permet d'y répondre pleinement, en délimitant les paramètres qui jouent un rôle dans la détermination de la référence, à savoir l'énonciation du nom et le contexte dans lequel cet acte a lieu (ce que représentent les variables $N$ et $S$ de la formulation du sens du nom propre que nous donnons plus haut).

D'ailleurs Kripke n'est pas totalement fermé à une telle théorie, pour laquelle il suggère même implicitement quelques précisions et aménagements. L'un des problèmes qu'elle présente est de rester descriptive ; Kripke souligne ainsi que «de quelque façon qu'on analyse cette relation "être appelé", c'est elle qui détermine la référence, et non une description comme "l'homme appelé 'Socrate"” " (1972:57). Notons que c'est là l'une des raisons principales qui ont fait revenir Kleiber sur son hypothèse initiale du prédicat de dénomination et lui ont fait finalement adopter la thèse d'un sens dénominatif procédural (voir Kleiber, 1995 et 2004).

\begin{abstract}
Nous avons là une théorie de la référence des noms propres. "Socrate » signifie simplement «l'homme appelé "Socrate" ». Peut-être, à vrai dire, n’y avait-il pas qu'une seule personne appelée "Socrate », et peut-être était-elle appelée « Socrate » par certains et non par d'autres. Mais on a là une condition qui, dans certaines circonstances, est satisfaite par un individu et un seul. Peut-être un seul homme était-il appelé « Socrate » par moi en une certaine occasion. (Kripke, 1972:56)
\end{abstract}

Il est tentant de voir ici une manière de souligner le fait que la référence du nom propre est relative aux sujets qui l'emploient et aux conditions dans lesquelles ils l'emploient, en particulier dans les derniers mots de cette citation où Kripke semble ménager une possibilité de validation empirique de la théorie : appelé "Socrate» par moi en une certaine occasion, ce segment a exactement la structure de la formulation du sens dénominatif que nous donnons plus haut.

Kripke semble donc admettre du bout des lèvres qu'une théorie du sens dénominatif puisse expliquer la référence du nom propre, mais le fait que l'explication qu'elle fournit ait un aspect circulaire reste pour lui un problème incontournable. Il nous semble, quant à nous, que ce problème n'en est pas un, ce que nous allons maintenant montrer en intégrant les paramètres de l'acte de nomination et des chaînes de communication à la représentation que nous sommes en train de dresser du sens des noms propres.

\title{
3.2 Dialogisme du nom propre
}

Kleiber s'est également heurté, en élaborant sa théorie du sens dénominatif des noms propres, au problème de la circularité de l'explication de la signification et de la récursivité de la définition du nom 
propre. La définition du sens du nom propre par l'instruction de "chercher le $x$ appelé $N$ " pose en effet le problème du statut attribué au terme $N$. Kleiber l'a clairement formulé dans le cadre de sa théorie du prédicat de dénomination, mais le problème reste le même dans le cadre de la théorie du sens dénominatif procédural qu'il lui substitue ensuite.

On peut arguer, en effet, que si Paul correspond à être appelé /Paul/(x), l'élément Paul de cette formule correspond également à être appelé /Paul/(x), l'élément Paul de cette dernière définition correspond aussi à être appelé $/ P a u l /(x)$, et ainsi de suite. On se trouve ainsi entraîné dans une régression à l'infini. Cette objection n'est cependant valide que si l'on accorde le statut de nom propre à la forme $N$ de être appelé $/ N /(x)$. Si $N$ ne représente pas un nom propre, on ne saurait évidemment lui substituer la définition être appelé $/ N /(x)$. (Kleiber, $1981: 342-343$ )

Kleiber considère d'abord que le terme $N$ ne représente pas le nom propre mais « la chaîne phonique ou graphique elle-même » $(1995: 12,1981: 399)$, mais il s'interroge encore en 2004 sur son statut en constatant que cette «question du statut du $\mathrm{N}$ qui figure dans le prédicat de dénomination [...] s'avère être en effet un obstacle insurmontable » (2004: 121). L'approche adoptée par Kleiber dans son examen de la question pose deux problèmes que nous souhaiterions ici relever.

Premièrement, il aborde la question du statut de $N$ dans la formulation du sens dénominatif en partant de l'analyse des énoncés de nomination didactique du type $\mathrm{Je} m$ 'appelle $N$, qui représentent un emploi typique du nom propre, et ce faisant il assure que $N$ ne doit pas avoir le même statut dans un énoncé de nomination et dans la formulation du sens dénominatif.

Deuxièmement, il s'oblige à trouver une solution spécifique pour caractériser le sens d'un nom propre actualisé dans un énoncé de nomination, arguant que, dans ce type d'emploi, il « ne fonctionne pas référentiellement, c'est-à-dire n'a pas le porteur du nom comme référent» (2004:121). Or, ce fonctionnement spécifique du nom propre dans les énoncés de nomination ne justifie pas à nos yeux un traitement différent du nom propre en tant que tel, dans la mesure où la valeur du nom est alors déterminée en grande partie par la syntaxe de l'énoncé qui actualise ce nom et par la sémantique du verbe s'appeler. La construction avec le verbe s'appeler donne cependant au nom propre une valeur autonymique, qui se retrouve également dans la formulation du sens dénominatif ; $N$ n'en reste pas moins un nom propre - en emploi autonymique si l'on veut - aussi bien dans les énoncés de nomination que dans le sens dénominatif.

Mais le principal problème, qui conduit Kleiber à distinguer le statut de $N$ dans les énoncés de nomination et dans la formulation du sens dénominatif, est qu'affirmer que $N$ représente un nom propre dans “chercher le $x$ appelé (ou qui s'appelle) $N$ " peut conduire à une régression à l'infini dans l'interprétation du nom propre. Autrement dit, si le terme $N$ est un nom propre, il doit lui-même être déterminé par l'instruction de "chercher le $x$ appelé $N$ ". Or, cela ne nous paraît pas contradictoire avec ce que nous avons vu de la théorie de la nomination de Kripke, au contraire.

Une question se pose néanmoins : dans quel contexte chercher la détermination du $N$ contenu dans le sens dénominatif? La théorie des chaînes causales permet de répondre à cette question : la détermination de $N$ est à chercher dans les énoncés qui forment la chaîne de communication reliant l'énoncé actuel à l'acte de nomination ; autrement dit la formule " $x$ appelé $N$ " est une représentation schématique de la valeur du nom dans un énoncé autre, et c'est précisément ce que représente ce sens dénominatif : un renvoi à un ou plusieurs énoncés logiquement antérieurs. Le rôle de la composante dénominative de ce sens est justement de rendre compte de ce caractère dialogique du nom propre. La notion de s'appeler ou être appelé ou encore porter le nom de présuppose un sujet qui appelle ou emploie le nom, et plus largement elle présuppose un usage (plus ou moins) répandu. C'est là que réside la dimension dialogique du nom propre : l'énonciation actuelle du nom propre renvoie à des énonciations autres, et ce renvoi détermine la référence du nom propre. Concrètement, l'individu que je dénomme $N$ est appelé ainsi par d'autres et est initialement nommé ainsi, ce qui explique - et même cause, dans la perspective des chaînes causales mon propre emploi. 
Il arrive cependant régulièrement que le référent du nom ne soit pas le porteur de ce nom. La détermination de la référence n'en suit pas moins le même chemin : si je dis c'est un petit Napoléon pour désigner tel individu qui porte en fait le nom de Martin, c'est en référence à certains discours, qui associent telles et telles propriétés au nom Napoléon (par exemple, en référence à une biographie que j'aurais lue, mais aussi à ce que j'ai appris en cours d'histoire, au contenu de conversations diverses que j'ai eues avec diverses personnes où le nom de Napoléon était employé, etc.). Reconnaissant ces propriétés chez Martin je décide, par jeu si l'on veut, de l'identifier à Napoléon, ou plus précisément de le qualifier de Napoléon. La compréhension d'un tel énoncé est relativement complexe puisqu'elle fait intervenir plusieurs discours différents, ainsi que la capacité des interlocuteurs à se représenter leurs états mentaux mutuels. Mais, en définitive, la référence et la signification du nom propre est alors déterminée par l'identité des partenaires de l'échange verbal et le contexte dans lequel il a lieu, qui comprend aussi les divers discours que draine l'énoncé, c'est-à-dire auxquels l'énoncé répond implicitement ou explicitement.

La récursivité de la définition n'est donc pas un problème; c'est au contraire une manifestation du caractère dialogique du nom propre. On a vu en effet que l'énonciation actuelle d'un nom propre fait référence à une énonciation antérieure de ce nom, qui elle-même fait référence à une énonciation antérieure, et ainsi de suite. On ne régresse cependant pas ainsi jusqu'à l'infini mais jusqu'à l'acte de nomination qui a institué l'usage de ce nom pour désigner tel individu. Précisons pour conclure qu'une telle représentation du sens du nom propre doit faire figurer, en plus de la composante dénominative, le paramètre contextuel; c'est pour cela que nous avons ajouté plus haut la variable $S$ à celles du référent $x$ et du nom $N$, l'élément de stabilité de la formulation du sens résidant dans sa composante dénominative, c'est-à-dire dans la relation entre les trois variables $x, N$ et $S$.

Cette solution présente toutefois un paradoxe. Il est en effet légitime de se demander ce que signifie le nom dans l'énoncé performatif de nomination. Or on se trouve alors dans une configuration où il n'existe pas d'énonciation antérieure où le terme $N$ aurait pu être employé, puisqu'on a alors précisément affaire à l'énoncé qui donne existence à ce terme comme nom propre. Le paradoxe est donc le suivant : on ne peut expliquer le sens du terme $N$ dans l'acte de nomination autrement qu'en disant que c'est précisément cet acte qui lui donne son sens. En d'autres termes, on a alors atteint le bout de la chaîne de communication, et c'est de cette manière qu'on résout le problème de la récursivité du sens du nom propre.

La nomination est cependant une pratique trop courante pour qu'un nom nouveau soit créé à chaque fois ; le nom est en général puisé dans un stock de formes disponibles. Lorsqu'on choisit un nom pour accomplir un acte de nomination on s'inspire en effet des pratiques existantes, on pioche parmi les noms qui appartiennent déjà à une et même plusieurs chaînes de communication et qui circulent déjà au sein d'un réseau de locuteurs. La motivation du nom propre peut ainsi être considérée comme expliquant le sens du terme $N$ dans l'énoncé performatif de nomination, et même le sens de l'acte de nomination luimême. Mais on se situe alors à un autre niveau : la dimension dialogique du nom propre est rompue en ce qui concerne la référence et le rapport au porteur du nom, mais elle se perpétue dans les connotations associées au nom. On peut ainsi espérer que le Titanic II connaîtra un meilleur sort que son illustre modèle, mais on peut difficilement ignorer que le drame que ce dernier a imprimé dans ce nom a largement éclipsé la grandeur divine qu'il était censé évoquer.

\section{Références bibliographiques}

Arrivé, M., Gadet, F., Galmiche, M. (1986). La grammaire d'aujourd'hui : guide alphabétique de linguistique française. Paris : Flammarion.

Austin, J. L. (1962). Quand dire c'est faire. Trad. de G. Lane. Paris : Éditions du Seuil, 1970.

Bakhtine, M. (1979). Les genres du discours. Trad. de A. Aucouturier. In Esthétique de la création verbale. Paris : Gallimard, 1984, 263-308.

Bakhtine, M. (1975). Du discours romanesque. Trad. de D. Olivier. In Esthétique et théorie du roman. Paris: Gallimard, 1978, 83-233. 
Benveniste, É. (1958). De la subjectivité dans le langage. In Problèmes de linguistique générale I. Paris : Gallimard, $1966,258-266$.

Cormier, A. (2011). La personne du nom propre : interprétation en contexte. In Bernard-Barbeau G., Gagné C., Leblanc G. (éds), Actes des XXIV Journées de linguistique, 4-5 mars 2010, Québec : CIRAL, 23-38.

Cormier, A. (2013a). Entre subjectivité et historicité, le rôle du nom propre dans la construction de l'identité personnelle. Interrogations, 16. [En ligne : http://www.revue-interrogations.org/Entre-subjectivite-et-historicite]

Cormier, A. (2013b). Rôle de l'énonciation dans l'analyse linguistique du nom propre. Limoges : Lambert-Lucas.

Frege, G. (1892). Sens et dénotation. Trad. de C. Imbert. In Écrits logiques et philosophiques. Paris : Éditions du Seuil, 1971, 102-126.

Gary-Prieur, M.-N. (1994). Grammaire du nom propre. Paris : Presses universitaires de France.

Gouvard, J.-M. (1998). La pragmatique : outils pour l'analyse littéraire. Paris : Armand Colin

Jonasson, K. (1994). Le nom propre : Constructions et interprétations. Louvain-la-Neuve : Éditions Duculot.

Kerbrat-Orecchioni, C. (2001). Les actes de langage dans le discours : Théorie et fonctionnement. Paris : Armand Colin, 2008.

Kleiber, G. (1981). Problèmes de référence : descriptions définies et noms propres. Paris : Klincksieck.

Kleiber, G. (1995). Sur la définition des noms propres : une dizaine d'années après. In Noailly M. (éd.), Nom propre et nomination: Actes du colloque de Brest, 21-24 avril 1994, Paris : Klincksieck, 11-35.

Kleiber, G. (2004). Peut-on sauver un sens de dénomination pour les noms propres ? Functions of Language, 11/1, $115-145$.

Kneale, W. (1962). Modality De Dicto and De Re. In Nagel E., Suppes P., Tarsky A. (eds), Logic, Methodology and the Philosophy of Science : Proceeding of the 1960 International Congress, Stanford : Stanford University Press, 622-633.

Kripke, S. (1972). La logique des noms propres. Trad. de P. Jacob et F. Recanati. Paris : Les Éditions de Minuit, 1982.

Lyons, J. (1977). Éléments de sémantique. Trad. de J. Durand. Paris : Larousse, 1978.

Neveu, F. (2004). Dictionnaire des sciences du langage. Paris : Armand Colin.

Riegel, M., Pellat, J.-C., Rioul, R. (2009). Grammaire méthodique du français, $4^{\mathrm{e}}$ édition. Paris: Presses universitaires de France.

Russell, B. (1948). La connaissance humaine : sa portée et ses limites. Trad. de N. Lavand. Paris : J. Vrin, 2002.

Saussure, F. de (2002). Écrits de linguistique générale. Paris : Gallimard.

Searle, J. R. (1969). Les actes de langage. Trad. de H. Pauchard. Paris : Hermann, 1972.

Searle, J. R. (1983). L'Intentionalité : Essai de philosophie des états mentaux. Trad. de C. Pichevin. Paris : Les Éditions de Minuit, 1985.

Siblot, P. (1997). Nomination et production de sens : le praxème. Langages, 31 : 127, 38-55.

Wittgenstein, L. (1953). Recherches philosophiques. Trad. de F. Dastur, M. Élie, J.-L. Gautero, D. Janicaud et É. Rigal. Paris : Gallimard, 2004

\footnotetext{
${ }^{1}$ Ou de l'homonymie des différents porteurs d'un même nom propre, mais ce n'est pas le lieu ici d'entrer dans le détail de ce problème.

${ }^{2}$ Naming and necessity.

${ }^{3}$ Kripke considère que les noms communs tels que tigre, or ou vache « sont beaucoup plus semblables aux noms propres qu'on ne le suppose ordinairement » (1972:115). Ces noms sont en effet pour lui des désignateurs rigides car ils servent davantage à désigner les individus qu'à décrire les traits caractéristiques qui permettent de les identifier comme appartenant à telle espèce. Ainsi le sens du nom tigre, par exemple, ne peut donner les conditions nécessaires
} 
et suffisantes, connaissables a priori, qu'un objet doit remplir pour appartenir à l'espèce des tigres, car ces propriétés sont empiriques et donc susceptibles d'évoluer avec le progrès des connaissances.

${ }^{4}$ Sur la distinction entre nomination performative et nomination didactique, qui distingue en gros l'énoncé qui établit de celui qui enseigne un nom propre, voir Lyons (1977 : 177).

${ }^{5}$ Pour un aperçu de la théorie des actes de langage, voir Benveniste (1958), Austin (1962), Searle (1969) et KerbratOrecchioni (2001). 